$\stackrel{W}{N}$

Global burnals Inc.

है

\title{
A Case Study of a Prosthetic Knee Joint Infection Following Periodontal Debridement
}

By Sabrina P. Heglund

University of Alberta

Abstract- This case study paper is written to raise awareness of prosthetic joint infection (PJI) of the right knee following periodontal debridement. The author understands that antibiotic prophylaxis for patients after the two-year post-operative milestone has been a topic of debate for over three decades. However, it is not like winning a lottery when a patient is in the small $2 \%$ of the knee prosthetic surgical population which develops a PJI. When prophylactic antibiotics and resistance to them are compared to the actual incidence of the PJI itself, costs cannot be restricted to the healthcare system, the source of income, and lack of mobility during recuperation, but should consider the patient's quality of life and the state of mind during recuperation.

Keywords: antibiotic prophylaxis, periodontal debridement, prosthetic joint infection, hematologic bacteria seeding, oral microbiota.

GJMR-H Classification: NLMC Code: WE 304

Strictly as per the compliance and regulations of:

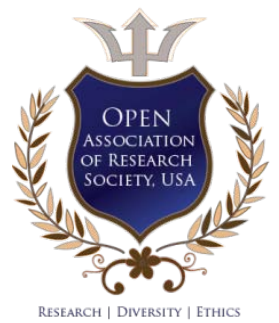

(C) 2019. Sabrina P. Heglund. This is a research/review paper, distributed under the terms of the Creative Commons AttributionNoncommercial 3.0 Unported License http://creativecommons.org/licenses/by-nc/3.0/), permitting all non-commercial use, distribution, and reproduction in any medium, provided the original work is properly cited. 


\title{
A Case Study of a Prosthetic Knee Joint Infection Following Periodontal Debridement
}

\author{
Sabrina P. Heglund
}

\begin{abstract}
This case study paper is written to raise awareness of prosthetic joint infection (PJI) of the right knee following periodontal debridement. The author understands that antibiotic prophylaxis for patients after the two-year postoperative milestone has been a topic of debate for over three decades. However, it is not like winning a lottery when a patient is in the small $2 \%$ of the knee prosthetic surgical population which develops a PJI. When prophylactic antibiotics and resistance to them are compared to the actual incidence of the PJI itself, costs cannot be restricted to the healthcare system, the source of income, and lack of mobility during recuperation, but should consider the patient's quality of life and the state of mind during recuperation.
\end{abstract}

Keywords: antibiotic prophylaxis, periodontal debridement, prosthetic joint infection, hematologic bacteria seeding, oral microbiota.

\section{INTRODUCTION}

A ntibiotic resistance has been on the rise and has lead to a paucity of antibiotics remaining to fight bacterial infections (Davies \& Davies, 2010). All healthcare personnel has been alerted to this fact of preventive medicine, and many guidelines have been arduously researched and published (Little, Jacobson, \& Lockhart, 2010). In the case of antibiotic coverage for possible hematological seeding from dental work, patients with artificial joints were given 3 grams of amoxicillin preoperatively for the first two years after the joint was replaced (Seymour, Whitworth, \& Martin, 2003). After that, guidelines agreed to by oral medicine practitioners and orthopedic surgeons, recommend the practice be restricted to high- risk patients as the risk for infections in knee replacement surgeries were reduced to $2 \%$ of patients receiving dental care (Zimmerli, Trampuz, \& Ochsner, 2004). Even in that case, the dental practitioner needed to converse with the orthopedic surgeon and ask for their specific recommendation for the patient they were about to treat. Three years after three monthly reappointments for dental hygiene therapy did not result in a knee infection, this patient was not prescribed antibiotics for his dental hygiene appointment. However, he recently had several gingival surgeries to reduce an oroantral communication from a previous tooth extraction and antibiotics were prescribed post-operatively each time surgery was done. The patient then had this periodontal

Author: Sabrina P. Heglund, Ph.D., University of Alberta, Edmonton, Alberta, Canada. e-mail: sheglund@ualberta.ca debridement therapy administered without antibiotics as the current guidelines (Sollecito et al., 2015) suggest, and eight days later succumbed to a prosthetic joint infection (PJI) of the right knee.

The right knee was initially entered in 2011 for total knee arthroplasty (TKA) due to the damage caused by osteoarthritis. In 2015 the patient recalled having severe pain in the right knee and after much physiotherapy was of no help, was returned to the orthopedic surgeon. The diagnosis was that a component of the TKA had worked loose and needed to be repaired. This reparative surgery resulted in an additional 6-8 week loss of wages as was the case after the initial surgery in 2011. The patient had received great relief in the right knee, so he had the left knee corrected with a TKA in 2012 with the same 6-8 week period of not working while recuperating and receiving physiotherapy. The left TKA has not caused any problems over the six years it has been in place. The 69-year-old male patient contracted bacteremia in the right knee and is presently following the orthopedic surgeons gold standard (Petretta, Phillips, \& Toms, 2017) of treatment which entails a two-stage treatment of the joint. The first stage is the removal of all hardware and the insertion of a temporary antibiotic cement spacer, and the second stage is re-entry for the placement of the final prosthesis.

\section{il. Method of Diagnosis}

The patient found the right knee swollen and extremely painful eight days after the completion of periodontal debridement. It would not bear weight, and the purchase of a set of crutches was needed to attend a medical doctor. Radiographs of the right knee showed inconclusive results of infection or further bone fractures. The physician discussed the history of the right knee with the patient and tentatively asked if he had any dental treatment recently. The patient replied that he had a dental hygiene appointment a week prior which caused the doctor to suspect a bacterial infection, especially with the reported symptoms of swelling, heat, and discomfort on weight bearing. He suggested a course of antibiotics that the patient was to initiate immediately and if there was no relief noted in 48 hours, he was to present to the emergency department of a hospital. Within 32 hours, the pain was excruciating, or 10 on a scale of 10 with ten being more than the patient could tolerate. The patient then reported 
to the emergency department of the hospital where the orthopedic surgeon had originally carried out the TKA's.

The admitting physician in the emergency department took over four hours to attend to the patient and then agreed to call in the orthopedic surgeon. This wait took another two hours and immediately seeing the patient, the surgeon ordered a sterile aspiration kit. Many ounces of fluid were drained off, and one was earmarked for culture and sensitivity testing. The surgeon ordered several radiographs and the patient was admitted overnight with an intravenous (IV) delivery of Cefazalon, a multi- bactericidal cephalosporin antibiotic. Emergency surgery, a two-stage revision of the PJI to remove hardware, took place the next morning following all the protocols specific to that orthopedic surgeon. Four days later the culture identified Streptococcus mutans with the antibiotic now being changed to IV Ceftriaxone.

The male patient remained in the hospital for six days then discharged with a peripherally inserted central catheter "PICC" line inserted in his left arm. Outpatient IV antibiotic therapy would be provided for the next six weeks at a hospital closer to the patient's home. Over the next six weeks, blood would be drawn every week to check C-Reactive Protein (CRP) and D-Dimer levels. The patient was asked to return to the orthopedic surgeon at the 3.5-week post-surgery date for suture removal. After a twoweek antibiotic free period, now eight weeks post-surgery, the aspiration of fluid from the surgical area was taken under sedation, and another culture was grown. There was no growth from that culture, so oral antibiotics for a 6-week period was unnecessary. Finally, the date for the revision joint components to be inserted was set 2.5 months after the PJI. For 3.5-weeks after the PJl emergency surgery, the patient wore a knee brace full-time and restricted movement without a two wheeled walker for assistance. At that time, the dressing and sutures were removed. The patient was instructed to use the brace and walker or crutches outside the home to assist with weight bearing and to prevent falls that might breakdown the temporary spacer inserted in the joint.

\section{Iil. Results at 3.5 Weeks Post PJI Entry}

The patient was treated with opioids (hydromorphone, Gabapentin, Trazadone) for pain, acetylsalicylic tablets to prevent bloodclots, diclofenac for arthritis symptoms, and Tramadol for breakthrough pain post-surgery. He has been weaned off the opioids and uses Tylenol for the relief of discomfort as needed which is very rare. The brace was taken off twice a day, and knee bends were attempted. Antibiotic IV therapy occurred daily through the PICC line after his caregiver delivered him to the hospital. Weekly, PICC line dressings were changed and blood samples were collected. The patient reports very slight dizziness upon getting up to standing position, but little or no pain. His activities are limited and he needs care for the functions of daily living of life.

This male who is otherwise in good health and who walks his dog a mile everyday, works three to four days a week, rides a motorcycle in good weather, and putts around the acreage doing all the maintenance has been reduced to an armchair and bed for rest, and walking with a walker or a cane on stairs. Due to the need for a walker, a full-time caregiver assisted with his daily needs. The patient who has always kept his mind busy reading, doing crossword puzzles, and memorizing large tracts of literature for pleasure, seems to lack concentration. He is not getting pleasure out of the mind work and tends to sleep a great deal. The high point of the day was the outing to the IV therapy.

The dressing was taken off at the 3.5- week mark, and the wound had healed well. There is no discharge of any fluids at the site, and the surgeon is pleased with all the lab tests to date. The patient was instructed to use the brace and crutches when outdoors until eight weeks postsurgery. Blood tests were taken when the patient was off all antibiotics and aspiration of fluids was done at that time. If all results were clear, the replacement would be inserted 10 to 12 weeks after this emergency surgery was carried out. The patient is unable to drive or move very freely as a possible fall would complicate the integrity of the temporized joint. The PJl has added up to 5 months of unemployment, the need for a full-time caregiver and the loss of the pleasures of life.

\section{Discussion}

Medical science and biomechanical theory have progressed to allow replacements of knees and hips which function well for many years for patients diagnosed with degenerative arthritis. They provide the patient mobility without pain, and the opportunity to carry on with life as he/she sees fit. With the aging population, called baby boomers, there are many more patients presenting with prosthetic replacement joints in need of dental care that they have embraced and cared about until their golden years- any age after 65.

From the 1980s there has been concern that there was a possibility that dental treatment could induce hematological seeding of bacteria in cases of endocarditis in patients with implanted heart valves. From that point on, discussions have been ongoing between the American Heart Association, the dental associations, and the orthopedic associations. Dr. Little (Little et al., 2010) addressed the history of these discussions in great detail. Stated in this discussion was that in 2003, the American Association of Orthopedic Surgeons (AAOS) and the American Dental Association (ADA) came together and published guidelines that did not recommend the need for antibiotic prophylaxis (AP) to patients with joint implants 
prior to undergoing dental treatment as there was insufficient/conclusive evidence for the practice.

In 2009, orthopedic surgeons raised concerns about these prosthetic implants becoming infected from dental manipulations. The AAOS published an information statement: (https://www.aaos.org/AAOSNow/2009/May/ cover/ cover2/).

Comprehensive "information statement" puts patient safety first

In February 2009, the AAOS Board of Directors approved the release of the information statement "Antibiotic Prophylaxis for Bacteremia in Patients with Joint Replacements." The new statement asserts that "Given the potential adverse outcomes and cost of treating an infected joint replacement, the AAOS recommends that clinicians consider antibiotic prophylaxis for all total joint replacement patients prior to any invasive procedure that may cause bacteremia."

However, as Dr. Little (Little et al., 2010) shows, this statement was only an opinion, and was therefore not supported or underwritten by the ADA.

At present, guidelines from 2003 have been followed resulting in both the AAOS and the ADA agreeing that no AP be routinely provided to patients with prosthetic joint implants. Dental hygienists follow the rule that after two years post implant placement, AP is not required. There is no challenge to this guide as there are few if any scientific studies to prove otherwise. Additionally, the ethics involved to do a rigorous clinical trial study would be nightmarish. In the meantime, patients with implants undergo dental treatment without AP. This male patient succumbed to a PJI as he made up a part of the $2 \%$ of patients who are at risk for such an infection.

The rub in this whole issue is that the cost to this patient far outweighs evidence-based practice and far exceeds the level and quality of life to which he has become accustomed. The transport costs, the caregiver costs, the hospital costs, the surgeon costs, the lab testing costs, the daily IV costs, the additional pain medication and probiotic costs, the loss of income, the stress on the body and mind, all cost more than "just" a dollar value. We in health care have been taught to carry out evidence-based practice. We are responsible for doing good and not harming our patients. How do we bear the weight of suffering in this "unlucky" patient who happens to be in the $2 \%$ of the patients who will fall prey to PJI? It cannot be justified, but it needs attention. Attention to this issue can be achieved by healthcare workers registering the outliers and reading about the many costs incurred. Prosthetic revision surgery costs are possibly four times more than the original surgery for the joint implant.

\section{Conclusion}

This manuscript has been written to draw attention and raise awareness of the fact that even in evidencebased practice, there is always a chance for ill effects to a certain number of patients. A full medical and dental history updated at every appointment may take time away from the task at hand. In the grand scheme of things, that time may save the few patients at risk for PJI from having to undergo such costs. These case studies do not bear much weight as they are "rare" occurrences. But the suffering of this patient must not go unnoticed. A complete patient history of recent dental procedures done for this patient could have avoided the above outcomes. Prediction of the need for AP prior to dental procedures are difficult to determine when given strict guidelines. It is hoped that this case study will encourage conversations with the patient about the suggested guidelines and his/her preference for AP prior to undergoing dental treatment.

\section{References Références Referencias}

1. Davies, J., \& Davies, D. (2010). Origins and Evolution of Antibiotic Resistance. Microbiology and Molecular Biology Reviews. https://doi.org/10.1128/MMBR.000 16-10

2. Little, J. W., Jacobson, J. J., \& Lockhart, P. B. (2010). The dental treatment of patients with joint replacements: A position paper from the American Academy of Oral Medicine. Journal of the American Dental Association, 141(6), 667-671. https://doi.org/ 10.14219/jada.archive.2010.0255

3. Petretta, R., Phillips, J., \& Toms, A. (2017). Management of acute periprosthetic joint infection of the knee - Algorithms for the on call surgeon. Surgeon, 15(2), 83-92. https://doi.org/10.1016/j. surge.2016.06.001

4. Seymour, R. A., Whitworth, J. M., \& Martin, M. (2003). Antibiotic prophylaxis for patients with joint prostheses - Still a dilemma for dental practitioners. British Dental Journal. https://doi.org/10.1038/sj.bdj.4810352

5. Sollecito, T. P., Abt, E., Lockhart, P. B., Truelove, E., Paumier, T. M., Tracy, S. L.,Frantsve-Hawley, J. (2015). The use of prophylactic antibiotics prior to Dental procedures in patients with prosthetic joints: Evidencebased clinical practice guideline for dental practitioners-a report of the American Dental Association Council on Scientific Affairs. Journal of the American Dental Association. https://doi.org/10.1016/j. adaj.2014.11.012

6. Zimmerli, W., Trampuz, A., \& Ochsner, P. E. (2004). Prosthetic-Joint Infections. New England Journal of Medicine. https://doi.org/10.1056/NEJMra040181 